\title{
A modified Mach-Zehnder inferometer for the study of coherence of laser
}

P. K. Shukla, K. K. Sarangpani, S. Talwar, G. Purbia, S. Nakhe, et al.

P. K. Shukla, K. K. Sarangpani, S. Talwar, G. S. Purbia, S. V. Nakhe, H. S. Vora, "A modified Mach-Zehnder inferometer for the study of coherence of laser," Proc. SPIE 9664, Ninth International Topical Meeting on Education and Training in Optics and Photonics, 96642G (24 October 2005); doi: $10.1117 / 12.2207568$

SPIE Event: Ninth International Topical Meeting on Education and Training in Optics and Photonics, 2005, Marseille, France 


\section{Ref ETOP017}

\section{A Modified Mach-Zender Interferometer for the Study of Coherence Of Laser}

P K Shukla*, K K Sarangpani, S Talwar, G S Purbia, S V Nakhe, H S Vora

LSED , Center For Advanced Technology , Indore - 452013 (INDIA) - email : pks@cat.ernet.in

\section{Abstract}

The coherence property of the laser light is an important aspect for the understanding of the optical physics. A modified geometry of the Mach-Zender interferometer has been proposed for measurement of the coherence of the laser. The geometry of the mirrors has been arranged in such a way that the reversible shear interferometer and the Michelson interferometer, both the types of the interferometers are set-up in a single geometry. Using this interferometer, the spatial and the temporal coherence of the laser beam can be measured. The interferometer is simple in design and is useful in the computer assisted visual learning of the coherence at the college and university level education and teaching in optics.

Interferometry,Coherence, Optics

\section{Keywords}

\section{Summary}

The coherence property of the laser light is important in understanding of the laser loptics and its various applications in holography etc. The reversible shear interferometer1 provides the measurement of the spatial coherence and the Michelson interferometer measures the temporal coherence of the laser. The conventional young slit method for spatial coherence measurement is not suitable for large diameter laser beams and it also requires multiple measurements. In this paper, a modified geometry of Mach Zender interferometer has been reported which can be used for the spatial coherence in a single measurement as well as for the temporal coherence measurement of the laser beam. The interferometer can be easily used in the large diameter laser beams.

The schematic of the interferometer has been shown in the figure-1. Its mirror geometry is similar to the Mach Zender interferometer, the W1 and W2 are two wedges at 450 and mirror M3 is mounted on the translation stage. The incident laser beam is split at wedge

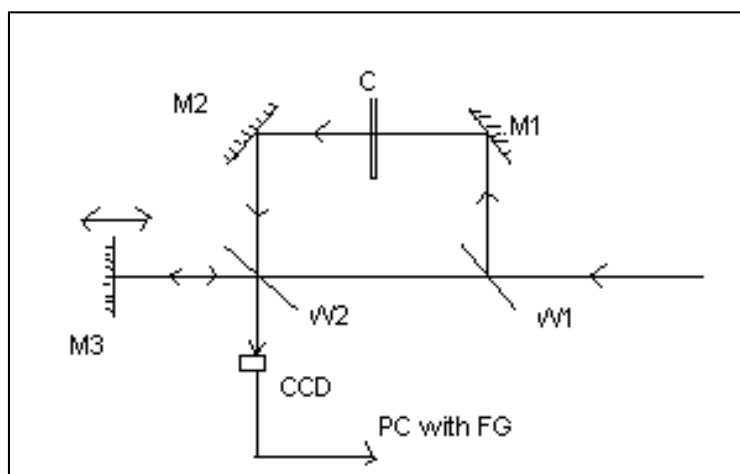

W1 and recombine at wegde W2. The one part of the laser beam propagating in the path $\mathrm{W} 1 \rightarrow \mathrm{M} 1 \rightarrow \mathrm{M} 2 \rightarrow \mathrm{W} 2$ and the other beam propagating along the path $\mathrm{W} 1 \rightarrow \mathrm{W} 2 \rightarrow \mathrm{M} 3 \rightarrow \mathrm{W} 2$, both the beams combine at wedge W2. C is the compensating plate. The wavefront from the earlier path is inverted along a line of folding.

Figure 1: Schematic of modified Mach Zender Interferometer 
The inverted wavefront interfere with other wavefront along a line of folding and the fringes are obtained by the tilt of the mirrors. The visibility of the fringes across the beam diameter gives the measure of spatial coherence in one dimension in a single measurement . Initially path length via M3 is kept same as path length via M2. For the measurement of the temporal coherence, the mirror M3 is moved apart such that visually the fringes vanishes from the position of the maximum visibility. The twice of the distance of the mirror movement of M3 gives the temporal coherence. Using this interferometer, the spatial coherence and the temporal coherence of typical unstable resonator output from the Copper Vapour Laser ( CVL ) has been measured and also the coherence studies on the multimode $\mathrm{He} \mathrm{Ne}$ laser has been carried out.

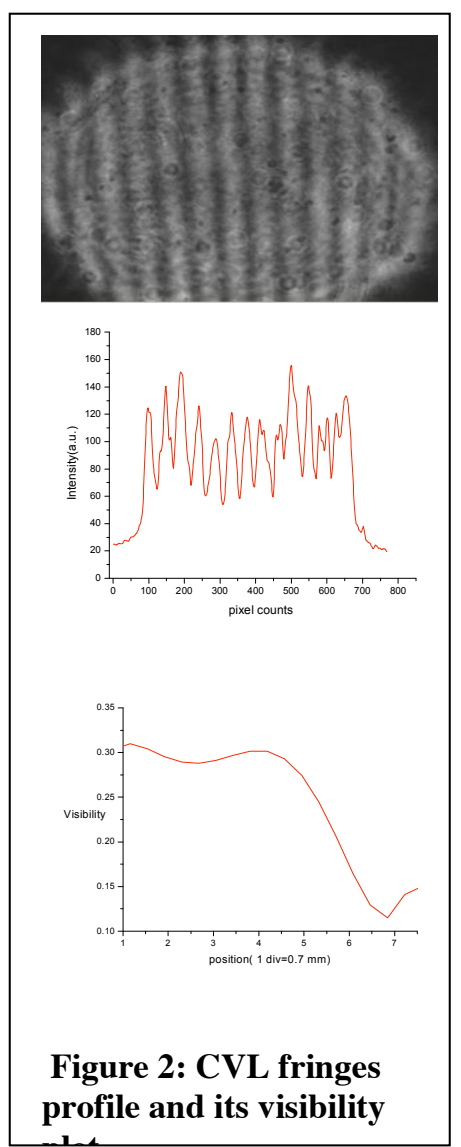

The interferogram can
be captured and
monitored on-line on
the PC with frame
grabber card and
compatible image processing software.

The reversible shear fringes for the CVL has been shown in the figure-2. The spatial coherence of the CVL is not good as can be visually observed from the interferogram. The spatial coherence width of the CVL (beam size $\sim 45 \mathrm{~mm}$ ) with typical uncollimated unstable resonator is approx. $8.0 \mathrm{~mm}$. The temporal coherence of the Copper vapour laser has been found to be $8 \mathrm{~cm}$. The shear fringes of the $\mathrm{HeNe}$ has also been shown in the figure-3. The temporal coherence of the $\mathrm{HeNe}$ laser has been found to be $\sim 15$
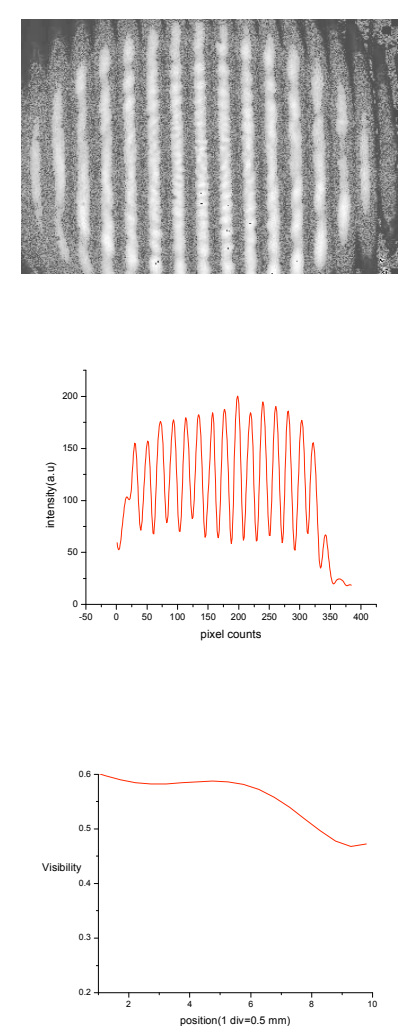

Figure 3: He-Ne fringes and its Visibility Curve

$\mathrm{cm}$. The HeNe has good spatial coherence across the beam diameter.The reduction in central fringe visibility from its actual value of 1.0 gives an qualitative presence of ASE (Amplified Spontaneous Emission) and also the multi mode nature of the laser beam. The interferometer can be easily set up in college optics laboratory and it will help in visual understanding and the measurement of the coherence of the laser beam. 


\section{Acknowledgement}

The authors are grateful to S S Kushwaha, D Shukla and I Satyanarayana for providing the the support for the operation of the CVL laser syetem.

\section{Reference}

1.T Omatsu, K.Kuroda, T Shimura, K Chihara, M Itoh \& I

Ogura,Opt.Quantum.Elec.23,S447 (1991)

Proc. of SPIE Vol. 9664 96642G-3

Downloaded From: https://www.spiedigitallibrary.org/conference-proceedings-of-spie on 26 Apr 2023 Terms of Use: https://www.spiedigitallibrary.org/terms-of-use 